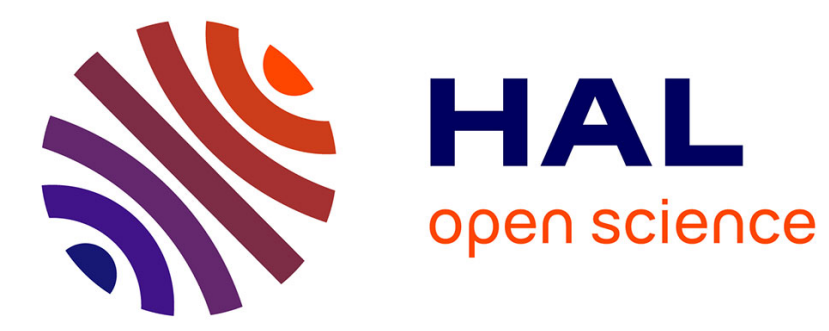

\title{
Stark broadening of high-members of the helium diffuse series in divertor plasmas
}

M Koubiti, H Capes, L Godbert-Mouret, Y Marandet, J Rosato, R Stamm

\section{To cite this version:}

M Koubiti, H Capes, L Godbert-Mouret, Y Marandet, J Rosato, et al.. Stark broadening of highmembers of the helium diffuse series in divertor plasmas. Journal of Physics B: Atomic, Molecular and Optical Physics, 2010, 43 (14), pp.144022. 10.1088/0953-4075/43/14/144022 . hal-00597862

\section{HAL Id: hal-00597862 \\ https://hal.science/hal-00597862}

Submitted on 2 Jun 2011

HAL is a multi-disciplinary open access archive for the deposit and dissemination of scientific research documents, whether they are published or not. The documents may come from teaching and research institutions in France or abroad, or from public or private research centers.
L'archive ouverte pluridisciplinaire HAL, est destinée au dépôt et à la diffusion de documents scientifiques de niveau recherche, publiés ou non, émanant des établissements d'enseignement et de recherche français ou étrangers, des laboratoires publics ou privés. 


\title{
Stark broadening of high-members of the helium diffuse series in divertor plasmas
}

\author{
M Koubiti, H Capes, L Godbert-Mouret, Y Marandet, \\ J Rosato and R Stamm \\ Laboratoire PIIM, UMR 6633 CNRS/Université de Provence, Centre Saint-Jérôme, \\ service 232, F-13397 Marseille Cedex 20, France \\ E-mail: mohammed.koubiti@univ-provence.fr
}

\begin{abstract}
Stark profiles of high-members $(\mathrm{n} \geq 7)$ of the helium triplet diffuse series $1 s 2 p^{3} P^{\circ}-1 s n d{ }^{3} D$ are calculated for tokamak divertor conditions using dipole reduced matrix elements obtained with a hydrogenic approximation. It is shown that the socalled "standard model" of Stark broadening is particularly suited to the description of the isolated lines $1 s 2 p^{3} P^{\circ}-1$ snd ${ }^{3} D$ with $n=8-10$. Applications to spectroscopic diagnostics in existing and future magnetic fusion-oriented devices like ITER are discussed.
\end{abstract}

PACS numbers: $32.30 . \mathrm{Jc}, 32.60+\mathrm{i}, 32.70 . \mathrm{Jz}$

Submitted to: J. Phys. B: At. Mol. Phys. 'Spectroscopic Diagnostics of Magnetic Fusion Plasmas' 


\section{Introduction}

In tokamaks with axisymmetric divertors, the partial or total detachment of the plasma from the targets in the divertor region is a consequence of charge-exchange and neutral collisional processes which lower the electron temperature and allow the ion-electron recombination processes to dominate the ionization processes. Under such conditions, recombination processes populate highly excited energy levels [i.e. with a high principal quantum number (PQN) n] of hydrogen/deuterium atoms, so that spectra of high-members of the hydrogen/deuterium Lyman, Balmer and Paschen series can be observed. Such spectra have been measured in several tokamaks, e.g. JET [1, 2], Alcator C-Mod [3, 4] and ASDEX Upgrade [5]. These spectra are commonly used for plasma electron density and temperature diagnostics. The electron temperature can be deduced spectroscopically from the measurements by several methods for instance those based on line intensities. However, the latter may require collisional-radiative modelling of level populations [6]. On the other hand, the electron density can be determined from the width or/and the shape of spectral lines for which Stark effect is the dominant broadening mechanism. The latter property has been previously applied to hydrogen emission on several occasions, e.g. in [7] where estimates of the electron density in the divertor of JET were obtained from line shape analysis. The idea proposed in the present paper is to extend this spectroscopic method to the high-members of the diffuse series of neutral helium. In fact, in its first phase, ITER should operate part of the time in helium, because of low activation requirements and lower H-mode threshold [8]. Similarly to high-n lines of hydrogen/deuterium, the He I $1 s 2 p{ }^{3} P^{\circ}-1 s n d{ }^{3} D$ triplet lines can be used for both electron density and temperature diagnostics in helium plasmas. They can also be used to check the consistency of the results extracted from hydrogen lines in the case of hydrogen/deuterium plasmas containing helium. Recently, neutral helium lines from high-lying levels have been observed and used for plasma diagnostic purposes in several fusion devices: for instance in a helium discharge with $D_{2}$ puffing in the JET divertor $[9,10,11,12]$ and in the linear divertor simulator NAGDIS-II [13, 14, 15, 16]. In the literature, articles dealing with Stark broadening of high- $n$ lines of neutral helium have been published by Griem and coworkers [17, 18, 19]. However, despite the large domain of electron density covered in these references $\left(10^{14}-10^{20} \mathrm{~cm}^{-3}\right)$, only ref. [19] concerns lines from upper PQNs $n \geq 5$, the others being limited to $n \leq 5$. The results were extended to higher values of $n$ for electron densities less than $10^{14} \mathrm{~cm}^{-3}$ using asymptotic formulae for line shapes at large separations from the line centers (line wings). Other papers [20,21], based on a semi-classical formalism of Stark broadening with both electrons and ions treated with the impact theory, present electron and ion broadening parameters of the He I diffuse series with $n \leq 10$ for electron densities of astrophysical interest. In tokamak divertors, the plasma conditions and the He I $1 s 2 p^{3} P^{\circ}-1 s n d{ }^{3} D$ triplet lines of interest may be different from those investigated by the above references.

The aim of this paper is to present an alternative approach, based on a hydrogenic 
approximation for the reduced dipole matrix elements, which is suitable to plasma diagnostics of tokamak divertor. The paper is organized as follows: In section 2, we present the underlying theory and the approximations on which is based the Stark line shape code (PPP [22]) used for the present calculations of line profiles. In section 3, we explain how the atomic data basis (especially the reduced dipolar matrix elements) is constructed in order to be used for line profile calculations of neutral helium. Results and discussions are reported in Sec. 4. Eventually, a conclusion is drawn in section 5.

\section{Formalism of Stark broadening}

The accuracy of a diagnostic based on Stark broadening of line emission depends on the simultaneous fulfilment of three conditions: the validity of the Stark broadening model, the accuracy of the used atomic data and the quality of the spectroscopic measurements (e.g. spectral or spatial resolution). The calculations presented in this paper do not account for gradient and density fluctuation effects. So we focus here on spectra of sufficiently high resolution which are obtained from homogeneous and stationary plasmas and hence discuss the first two conditions.

It is known that a spectral line emitted by an atom or an ion embedded in a plasma is affected by the electric micro-fields produced by the electrons and ions. The Stark broadening theory describes the effect of charged particles on line profiles. The so-called "standard model" [23], which we adopt here, is based on a semi-classical picture, i.e. the emitter is considered as a quantum system while the perturbers are treated classically using Newton's equations of motion. In the dipolar approximation, the profile $I(\Delta \omega)$ of a line is the one-side Fourier transform of the dipole autocorrelation function $C_{d d}(t)$ :

$$
I(\Delta \omega)=\frac{1}{\pi} \Re \int_{0}^{\infty} C_{d d}(t) e^{i \Delta \omega t} d t,
$$

where $\Delta \omega=\omega-\omega_{0}$ is the detuning from the unperturbed angular frequency $\omega_{0}$ of the line and $\Re$ stands for the real part of the integral. In the Liouville space (direct product of two Hilbert spaces), the autocorrelation function of the dipole can be expressed as follows:

$$
C_{d d}(t)=\left\langle\left\langle\mathbf{d}^{*}|\{U(t)\}| \rho_{0} \mathbf{d}\right\rangle\right\rangle,
$$

where $\mathbf{d}$ and $\rho_{0}$ are respectively the dipole and the density operator of the emitter, $U(t)$ represents the emitter evolution operator, and the brackets $\{\ldots\}$ denote an ensemble average over the states of the perturbers. The emitter evolution operator $U$ obeys the following stochastic Liouville equation:

$$
\frac{\partial U}{\partial t}=-i L U
$$

where the Liouvillian operator $L=L_{0}+l(t)$ is the sum of two terms $L_{0}$ and $l(t)$ denoting respectively the Liouvillian of the unperturbed emitter (including the fine structure term) and that of the time-dependent interaction of the emitter with the perturbers, 
i.e., the plasma electrons and ions. The latter may be written as follows: $l(t)=-\mathbf{d . F}(t)$ where $\mathbf{F}(t)$ is the electric field due to the plasma charged perturbers. Since electrons and ions in a plasma have typical velocities differing by more than an order of magnitude, their interactions with the emitter are characterized by different time scales. Naturally, this suggests a description based on the separation of the time scales of their interactions with the emitter. If $t_{s}=1 / \Delta \omega_{S}$ stands for the time of interest of the Stark broadening mechanism $\left(\Delta \omega_{S}\right.$ being the Stark width), the main contribution to the integral in Eq. (1) comes from interactions of duration smaller than $t_{s}$. The correlation time of the interaction of the emitter with ions $\tau_{i}$ is greater than the time of interest $t_{s}\left(\tau_{i}>>t_{s}\right)$. Conversely the correlation time of the interaction of the emitter with electrons $\tau_{e}$ is lower than $t_{s}\left(\tau_{e}<<t_{s}\right)$. This justifies the adoption of the common model which consists in assuming the ions static during $t_{s}$ ("quasi-static approximation") and the electrons as fast particles affecting the atom's radiation like a series of binary collisions ("impact approximation"). The line shape code PPP [22] we use for the present calculations relies on these two approximations.

The validity criterion of the impact approximation for electrons can be expressed in terms of the Weisskopf radius $\rho_{w e}$ and the mean distance between electrons $r_{e}$ as follows: $\rho_{w e} / r_{e}<<1$. The Weisskopf radius, also known as the strong collision radius, for a helium neutral at level $n \geq 7$ is taken equal to that of a hydrogen atom in level $n$, i.e. $\rho_{w e}=\hbar n^{2} / m_{e} v_{e}$ where $m_{e}$ and $v_{e}$ are respectively the mass and thermal velocity of a free plasma electron. This is justified by the hydrogen-like behavior of helium atoms at high $\mathrm{n}$ levels: the orbit of the excited electron of a neutral helium atom in the $n=7$-level is not greatly different from the one of the hydrogen orbits and the level energies are close to those of a hydrogen-like atom [24]. The mean interparticle distance is related to the plasma electron density by $r_{e}=\left(3 / 4 \pi N_{e}\right)^{1 / 3}$. Considering that $\rho_{w e} / r_{e}<0.1$ is sufficient for the impact approximation to be valid, we have plotted in Fig. $1, \rho_{w e} / r_{e}=0.1$ for different values of $n$ as a function of the plasma electron density and temperature. For each value of $n$, the impact approximation is valid in the region located above the corresponding iso-ratio curve $\left(\rho_{w e} / r_{e}=0.1\right)$, which is a straight line here because of the logarithmic scale. The domains of electron density $10^{12}-10^{15} \mathrm{~cm}^{-3}$ and temperature $0.03-1000 \mathrm{eV}$ shown in the figure largely cover the divertor conditions of magnetic fusion devices. According to Fig. 1, for an electron temperature $T_{e}>10 \mathrm{eV}$, the electron impact approximation is valid for several values of $n$ (including $n=8-12$ ) over almost the whole $N_{e}$. On the other hand, when $T_{e} \lesssim 1 \mathrm{eV}$, this approximation is valid for $n=8$ at $N_{e} \lesssim 10^{14} \mathrm{~cm}^{-3}$, for $n=9$ at $N_{e} \lesssim 6 \times 10^{13} \mathrm{~cm}^{-3}$, for $n=10$ at $N_{e} \lesssim 4 \times 10^{13} \mathrm{~cm}^{-3}$ and for $n>12$ at $N_{e}<10^{13} \mathrm{~cm}^{-3}$. It should be noted that setting for instance $\rho_{w e} / r_{e}<0.2$ instead of $\rho_{w e} / r_{e}<0.1$ as the criterion for the validity of the impact approximation extends the previous upper limits of the electron density to higher values for each electron temperature. In this case for $T_{e} \lesssim 1 \mathrm{eV}$ and electron densities up to $N_{e}=10^{14} \mathrm{~cm}^{-3}$ the impact approximation remains valid for $n \leq 12$. It should be noted that for given electron density and temperature, the validity of the 
impact approximation may be questionable for higher values of $n$.

In contrast to the impact approximation, the quasi-static approximation is valid in the opposite case, i.e. $\rho_{w e} / r_{e}>>1$. Similarly to electrons we have plotted in Fig.1 the ion curves $\rho_{w i} / r_{i}=0.1$ and $\rho_{w i} / r_{i}=1$ (thick lines), where $r_{i}$ and $\rho_{w i}$ are respectively the ion mean distance and the ion Weisskopf radius given by $r_{i}=\left(3 / 4 \pi N_{i}\right)^{1 / 3}$ and $\rho_{w i}=\hbar n^{2} / m_{e} v_{i}$. Here $N_{i}$ is the ion density and $v_{i}$ is the ion velocity. With respect to the impact approximation, it can be seen from Fig.1 that even for the most advantageous case corresponding to $n=8$ and an ion density of $10^{12} \mathrm{~cm}^{-3}$, this (impact) approximation is valid for ions with temperatures higher than about $200 \mathrm{eV}\left(T_{i} \geq 200 \mathrm{eV}\right)$. On the other hand, below the thick line representing the ion curve $\rho_{w i} / r_{i}=1$, which is close the electron curve $\rho_{w e} / r_{e}=0.1$, the ion Weisskopf radius $\rho_{w i}$ is greater than the ion mean distance $r_{i}$ and the quasi-static approximation can be used. In divertor regions of tokamaks and other fusion devices like stellarators, plasma densities and temperatures are in the ranges $\left(N_{e, i}=5 \times 10^{12}-10^{15} \mathrm{~cm}^{-3}\right.$ and $\left.T_{e, i}=1-100 \mathrm{eV}\right)$. For ions, these conditions correspond to regions below the curves $\rho_{w i} / r_{i}=1$ for all $n \geq 8$ (see Fig. 1 for $n=8$ ). Therefore we can use the quasi-static approximation to treat the interactions of the emitter with the plasma ions for all the He I $1 s 2 p{ }^{3} P^{\circ}-1$ snd ${ }^{3} D$ lines with $n \geq 8$. Note that when neither the quasi-static approximation nor the impact approximation is valid for ions, one can use more accurate approaches accounting for ion dynamics, e.g., the "frequency fluctuation model" which is already implemented in the PPP line shape code [22].

\section{Atomic data}

The Stark line shape code PPP [22] uses as an input data concerning both the emitter and the perturbers (plasma electrons and ions). Data concerning the emitter include energies, level populations and dipolar transition matrix elements. In this paper we are interested in He I emission lines resulting from transitions between triplet sublevels 1 snd ${ }^{3} D$ and $1 s 2 p{ }^{3} P^{\circ}$ with $n \geq 7$. Calculations of Stark profiles of He I triplet lines with a complete set of sublevels, (for each $n$, all sublevels of orbital quantum number $l \leq n-1$ are used) on the one side and a simplified set of sublevels (for each $n$, only sublevels with $l \leq 4$ are retained) on the other side, show no noticeable difference. We have therefore retained only the $1 s n l^{3} L$ energy sublevels with $7 \leq n \leq 20$ and $0 \leq l \leq 4$, i.e., sublevels 1 sns ${ }^{3} S$, 1 snp ${ }^{3} P^{\circ}$, 1 snd ${ }^{3} D$, 1 snf ${ }^{3} F^{\circ}$, and 1 sng ${ }^{3} G$ with $1 s 2 p^{3} P^{\circ}-1 s n s^{3} S$ and $1 s 2 p^{3} P^{\circ}-1 s n d^{3} D$ as the allowed transitions (see Fig. 2). Note that as the fine structure effect is negligible for high $\mathrm{n}$, all sublevels $1 s n l^{3} L_{J}$ corresponding to the possible values of the total momentum $J$ have the same energy as the

parent sublevel $1 s n l^{3} \mathrm{~L}$. It was mentioned previously that the use of Stark broadening for electron density diagnostic purposes requires atomic data as accurate as possible. Although energies of sufficient accuracy of neutral helium sublevels can be taken from the NIST database [25] for instance, many reduced matrix elements of dipole transitions 
from high- $n$ levels are unavailable. Therefore we have constructed a helium atomic data set where energies have been taken from NIST [25] while dipole matrix elements were computed using a hydrogenic approximation.

Fig. 2 shows the retained energy sublevels for each PQN $n$ and the allowed (solid arrows) and forbidden transitions (broken arrows). Sublevels corresponding to $n=8$ and their energy separations in $e V$ are illustrated on the left side of the figure. Based on hydrogenic radial wavefunctions, we have used formula (60.11), (63.2) and (63.3) from A. Bethe and E. Salpeter's textbbok [26] to calculate the reduced matrix elements $\left\langle 2 p{ }^{3} P^{\circ}\|\mathbf{d}\| n d^{3} D\right\rangle$ of the He I dipole transitions $1 s 2 p{ }^{3} P^{\circ}-1 s n d{ }^{3} D$. The use of the hydrogenic wavefunctions for neutral helium lines are easily justified in the case of single optical electron transitions from high- $n$ levels. Absolute values of the matrix elements $\left\langle 2 p^{3} P^{\circ}\|\mathbf{d}\| n d^{3} D\right\rangle$ for $1 s 2 p^{3} P^{\circ}-1 s n d^{3} D$ transitions with $n=4-20$, calculated with the hydrogenic approximation are shown by open circles $(O)$ in Fig. 3. They are compared to values deduced from transition probabilities given in references $[14,25]$. Note that in Fig. 3 of our previous paper [10], we have compared transition probabilities calculated using the hydrogenic approximation to those of refs. [14, 25]. The main discrepancies between A-values in Fig.3 of [10] are due to an error in the conversion of the hydrogenic dipole reduced matrix elements to transition probabilities (A-values) where a factor $2 S+1=3$ ( $S$ being the spin of the atom) was missing. This does not affect the data analysis results. As transition probabilities of the dipole transitions $1 s 2 p^{3} P^{\circ}-1 s n d^{3} D$ are not all available in the NIST data base [25] especially for high n, a power law was used in [14] to calculate the missing ones $A_{n^{3} D \rightarrow 2^{3} P^{\circ}}$ :

$$
A_{n^{3} D \rightarrow 2^{3} P^{\circ}}\left[10^{8} s^{-1}\right]=22.748 \times n^{-3.2396} .
$$

For a transition between an upper level $k$ and a lower level $i$, the transition probability $A_{k \rightarrow i}$ is related to the line strength $S^{\prime}(k, i) \equiv S_{k \rightarrow i}^{\prime}$ by the following:

$$
A_{k \rightarrow i}=\frac{2.0261 \times 10^{18}}{g_{k} \lambda^{3}} S_{k \rightarrow i}^{\prime},
$$

where $\lambda$ is the wavelength of the transition expressed in $\stackrel{\circ}{A}$ and $g_{k}$ is the statistical weight of the upper level $k$. In this relation atomic units are used for the line strength $S_{k \rightarrow i}^{\prime}$. The line strength $S^{\prime} \equiv S^{\prime}\left(n d^{3} D_{J_{k}}, 2 p{ }^{3} P_{J_{i}}^{\circ}\right)=\left|\left\langle 2 p^{3} P_{J_{i}}^{\circ}\|\mathbf{d}\| n d^{3} D_{J_{k}}\right\rangle\right|^{2}$ of a transition $2 p^{3} P_{J_{i}}^{\circ}-n d^{3} D_{J_{k}}$ is related to the dipole reduced matrix element $\left\langle 2 p^{3} P^{\circ}\|\mathbf{d}\| n d^{3} D\right\rangle$ by the following relation [27]:

$$
\left\langle 2 p^{3} P^{\circ}\|\mathbf{d}\| n d^{3} D\right\rangle=-a_{0} \sqrt{\frac{S^{\prime}}{2 S+1}}=-a_{0} \sqrt{\frac{S^{\prime}}{3}},
$$

where $S=1$ is the spin and $a_{0}$ is the Bohr radius. Here $J_{i}$ and $J_{k}$ are the total momentum values of the lower and upper levels respectively. The prime used for the line strength $S^{\prime}$ designation aim to prevent confusion with the spin $S$. One can see clearly that the matrix elements obtained from references $[14,25]$, which are shown in Fig. 3 by open triangles $(\triangle)$, are in a very good agreement with 
those calculated using the hydrogenic approximation. Following this, we use the same hydrogenic approximation to calculate the reduced matrix elements of all the allowed dipolar transitions including $\left\langle 1\right.$ sns ${ }^{3} S\|\mathbf{d}\| 1$ snp $\left.{ }^{3} P^{\circ}\right\rangle,\left\langle 1\right.$ snp ${ }^{3} P^{\circ}\|\mathbf{d}\| 1$ snd $\left.{ }^{3} D\right\rangle$, $\left\langle 1\right.$ snd ${ }^{3} D\|\mathbf{d}\| 1$ snf $\left.{ }^{3} F^{\circ}\right\rangle$ and $\left\langle 1\right.$ snf ${ }^{3} F^{\circ}\|\mathbf{d}\| 1$ sng $\left.{ }^{3} G\right\rangle$ with $7 \geq n \geq 20$.

\section{Results and discussion}

As attention is focused here on Stark broadening, the profiles to be discussed have been calculated without Zeeman and Doppler effects. Doppler line width of any of the considered He $1 s 2 p^{3} P^{\circ}-1 s n d^{3} D$ lines is less than $0.45 \stackrel{A}{A}$ for a neutral temperature of $10 \mathrm{eV}$ and even less than $0.15 \AA$ for a temperature of $\sim 1 \mathrm{eV}$. As we will see later below, for divertor plasma conditions, Doppler line widths are usually below the Stark line widths for the most interesting lines considered here. However, at the lowest electron densities, depending on the neutral temperature for instance, other broadening mechanisms may be of the same order or even dominate the Stark effect and have to be accounted for. Even though the PPP line shape code can easily deal with several broadening mechanisms (using for instance a convolution procedure), such situations are not considered in this paper. Details concerning the PPP code used for the calculations can be found in [22] and the references therein. For a cold plasma with a temperature $T_{e} \sim 1 \mathrm{eV}$, the validity of the impact approximation for electrons is fulfilled for transitions from $n=8-12$ for electron densities up to $N_{e} \sim 10^{14} \mathrm{~cm}^{-3}$ and even at higher densities for $n=8$ and $n=9\left(N_{e} \lesssim 5 \times 10^{14} \mathrm{~cm}^{-3}\right.$ for $\left.n=9\right)$.

As the $1 s 2 p^{3} P^{\circ}-1 s 11 d^{3} D$ and $1 s 2 p^{3} P^{\circ}-1 s 12 d^{3} D$ lines may be blended due to Stark effect, lines $1 s 2 p^{3} P^{\circ}-1 s n d^{3} D$ with $n=8-10$ appear to be the most convenient for density diagnostics of recombining plasmas like in tokamak divertors. The results of calculations concerning these lines are illustrated in Figs. 4, 5 and 6. The profiles have been calculated considering a deuterium plasma with an electron temperature of $1 \mathrm{eV}$. The calculations would not be different for a helium plasma in which $\mathrm{He}^{+}$ions are the majority. In Fig. 4, we show the $1 s 2 p^{3} P^{\circ}-1 s 8 d^{3} D$ line profiles calculated for electron densities of $10^{14} \mathrm{~cm}^{-3}$ (dashed line) and $3.5 \times 10^{14} \mathrm{~cm}^{-3}$ (dotted line). The line FWHM increases from $\sim 0.8 \AA$ at $N_{e}=10^{14} \mathrm{~cm}^{-3}$ to $\sim 1.8 \AA$ at $N_{e}=3.5 \times 10^{14} \mathrm{~cm}^{-3}$. Similarly, calculated profiles of the $1 s 2 p^{3} P^{\circ}-1 s 9 d^{3} D$ line are shown in Fig. 5 for the same plasma conditions, i.e. $N_{e}=10^{14} \mathrm{~cm}^{-3}$ (dashed line) and $N_{e}=3.5 \times 10^{14} \mathrm{~cm}^{-3}$ (dotted line). The FWHM of the lines increases from $\sim 1 \AA$ to $\sim 2.9 \AA$ when the density is enhanced from $N_{e}=10^{14} \mathrm{~cm}^{-3}$ to $N_{e}=3.5 \times 10^{14} \mathrm{~cm}^{-3}$. Note that the shoulders appearing in the red side of the two lines shown in Figs. 4 and 5 represent the forbidden components $1 s 2 p^{3} P^{\circ}-1 s 8 p^{3} P^{\circ}$ and $1 s 2 p^{3} P^{\circ}-1 s 9 p^{3} P^{\circ}$ due to the ion contribution of Stark effect.

The use of high- $n$ lines of neutral helium for plasma diagnostics may require data assessment. A possible way for that would be to compare Stark profiles calculated using the available atomic data with spectra composed of high- $n$ lines emitted simultaneously 
by deuterium/hydrogen and helium from the same plasma region, because atomic data for a hydrogenic emitter are known with a very good accuracy. Such deuterium/helium spectra can for example be obtained in divertor simulators, or in other plasma devices and tokamaks. Another way would consist in the use of independent electron density diagnostic methods. The electron density that can be deduced using these Stark broadened He I lines is a spatial (along the line of sight) and temporal average. It should be noted that if a drift wave turbulence exists along the line of sight, then the deduced electron density may contain a contribution from fluctuations and this generally leads to an overestimation of the electron density. Our group has recently started investigating the effects of fluctuations especially low-frequency turbulence on line shapes (see for instance [28]). Note that similarly to hydrogen lines in low-density in magnetized plasmas [29], it may be necessary to simultaneously account for Stark and Zeeman effects and to use the impact approximation for both ions and electrons.

\section{Conclusion}

Stark broadening of He I $1 s 2 p{ }^{3} P^{\circ}-1 s n d{ }^{3} D$ lines has been studied for conditions relevant to recombining plasmas of tokamak divertors. The study was focused on isolated members of the He I diffuse series corresponding to $n=8-10$ for which both the ion quasi-static and electron impact approximations hold over almost the considered electron density and temperature domains. An atomic data set was built using energies taken from the NIST database and hydrogenic radial wavefunctions for the calculation of the dipolar matrix elements of the He I $1 s 2 p{ }^{3} P^{\circ}-1 s n d{ }^{3} D$ transitions. The values of the subset of reduced matrix elements which are available in the literature are in good agreement with our calculations, showing that our method is reliable. For each of these lines $1 s 2 p^{3} P^{\circ}-1 s 8 d^{3} D, 1 s 2 p^{3} P^{\circ}-1 s 9 d^{3} D$, and $1 s 2 p^{3} P^{\circ}-1 s 10 d^{3} D$, Stark profiles were calculated using the PPP code for different plasma conditions. It is suggested to use these lines for plasma diagnostics of tokamak divertors as they are isolated and sensitive to Stark broadening, especially for ITER where helium discharge scenarios are foreseen [8].

\section{Acknowledgments}

This work was supported by the French Fédération de Recherche sur la Fusion par Confinement Magnétique FR-FCM, collaboration LRC DSM 99-14 (PIIM/CEA Cadarache) and the French Agence Nationale de la Recherche ANR Project "PHOTONITER" (Contract ANR-07-BLAN-0187-01). 


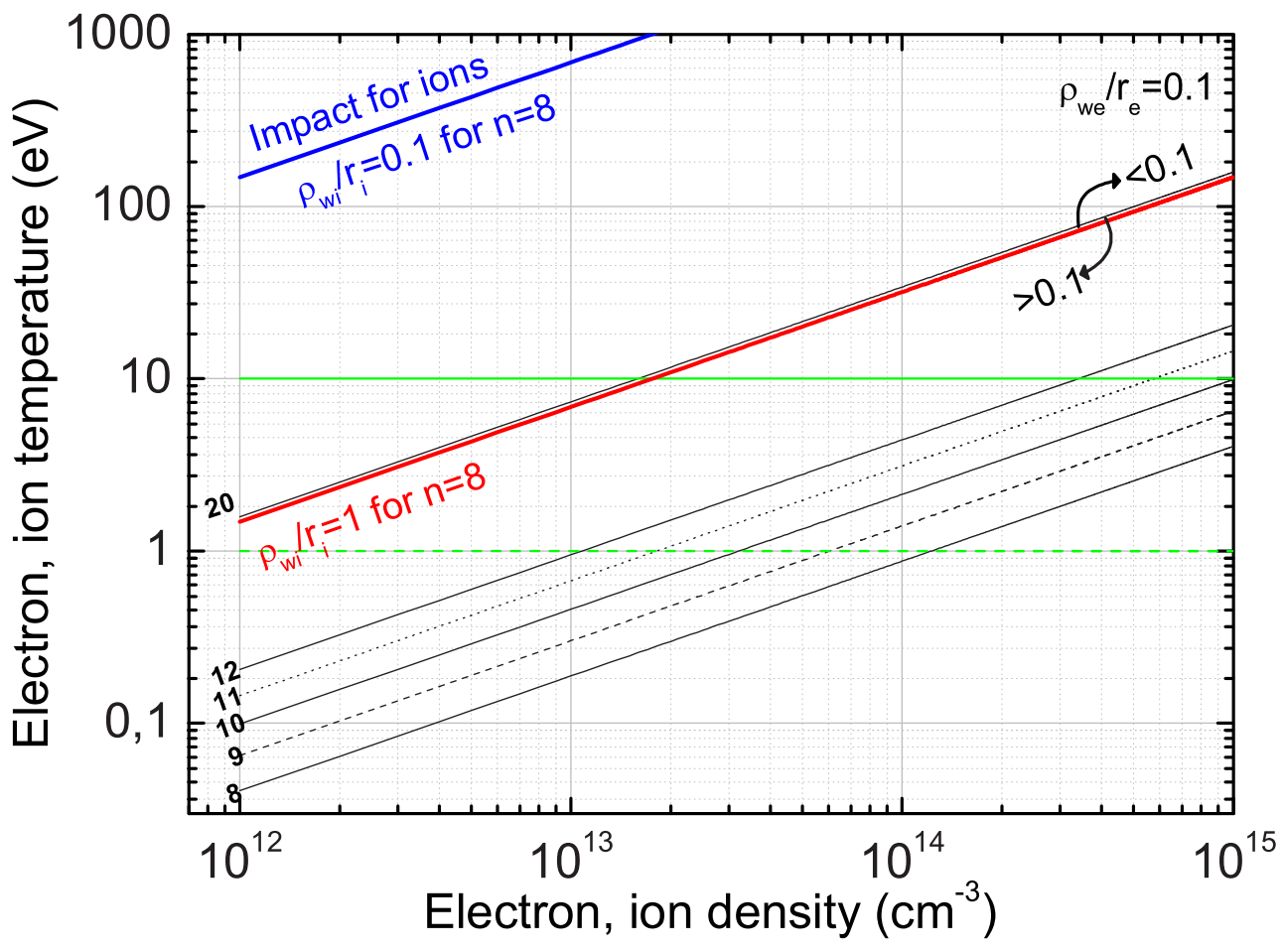

Figure 1. 
$\Delta \mathrm{E}\left(10^{-3} \mathrm{eV}\right)$ for $\mathrm{n}=8$
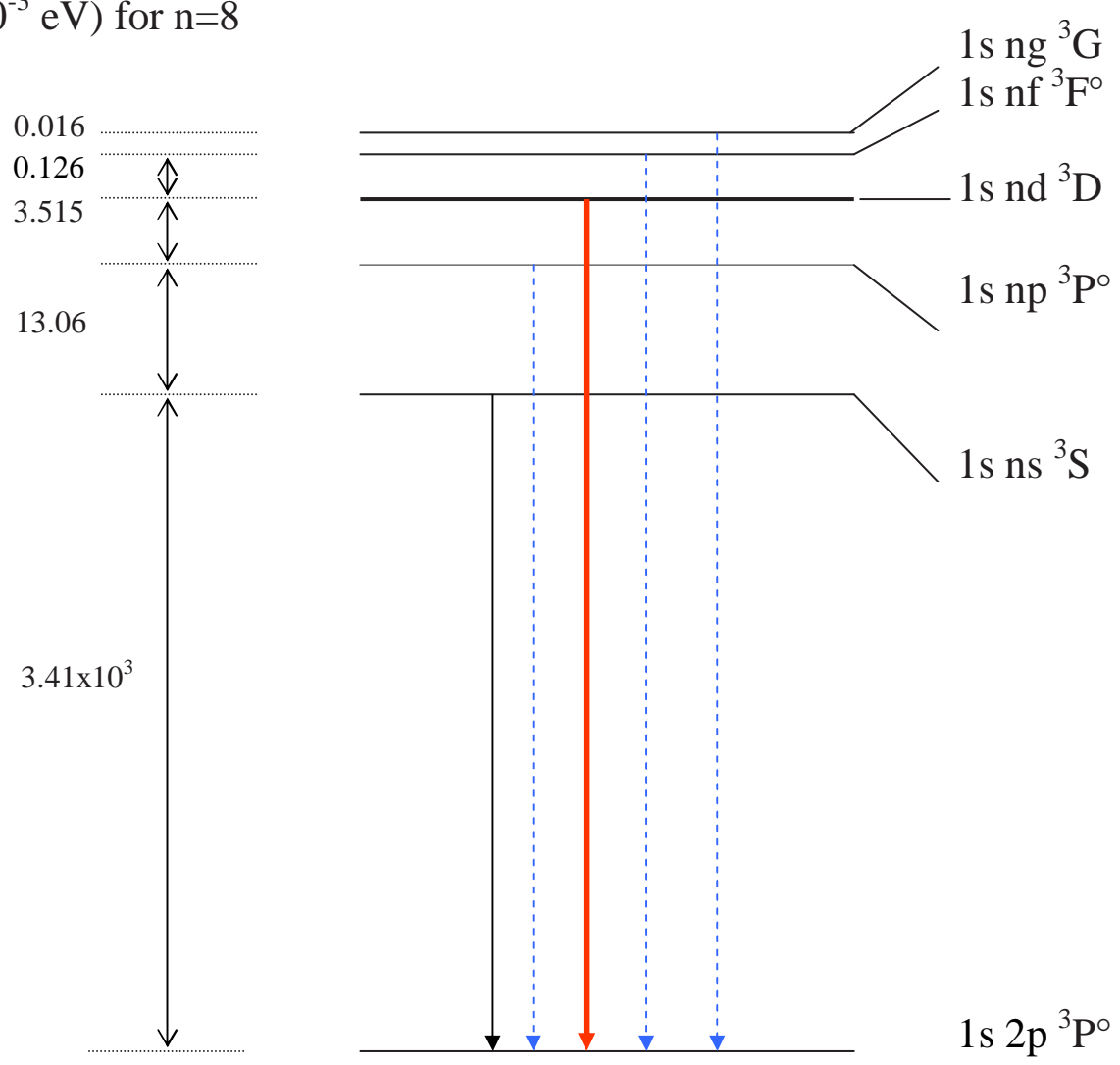

Figure 2. 


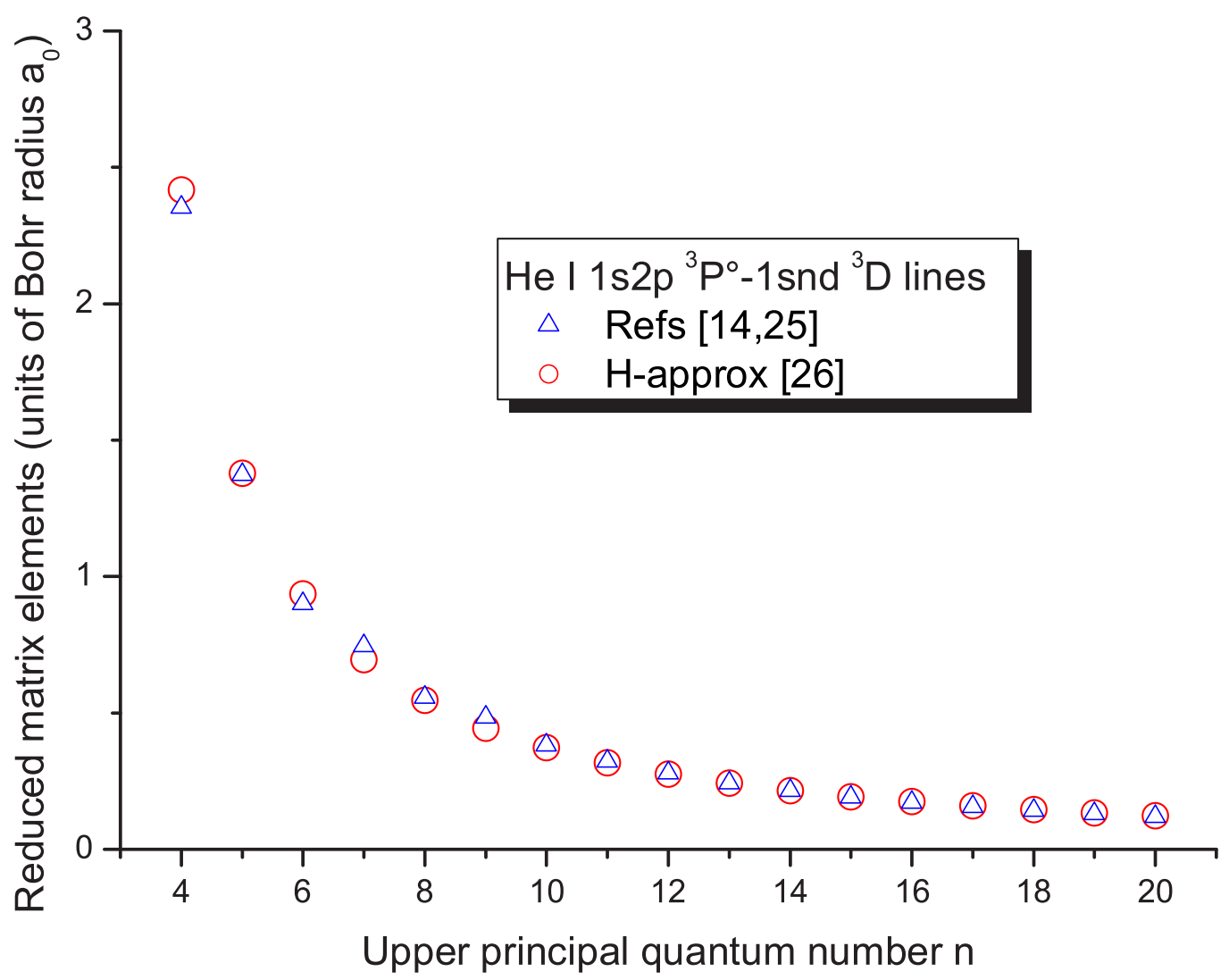

Figure 3. 


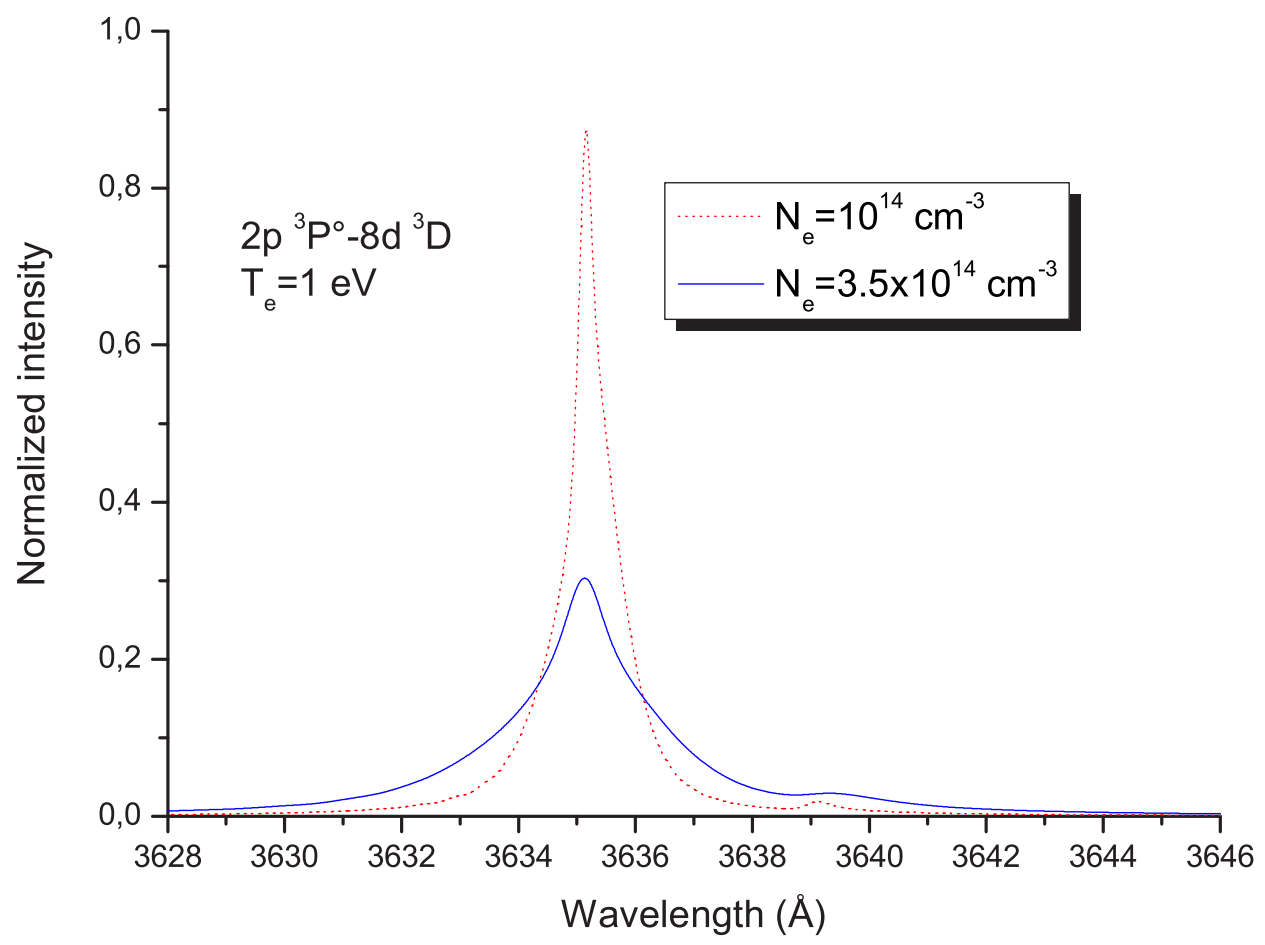

Figure 4. 


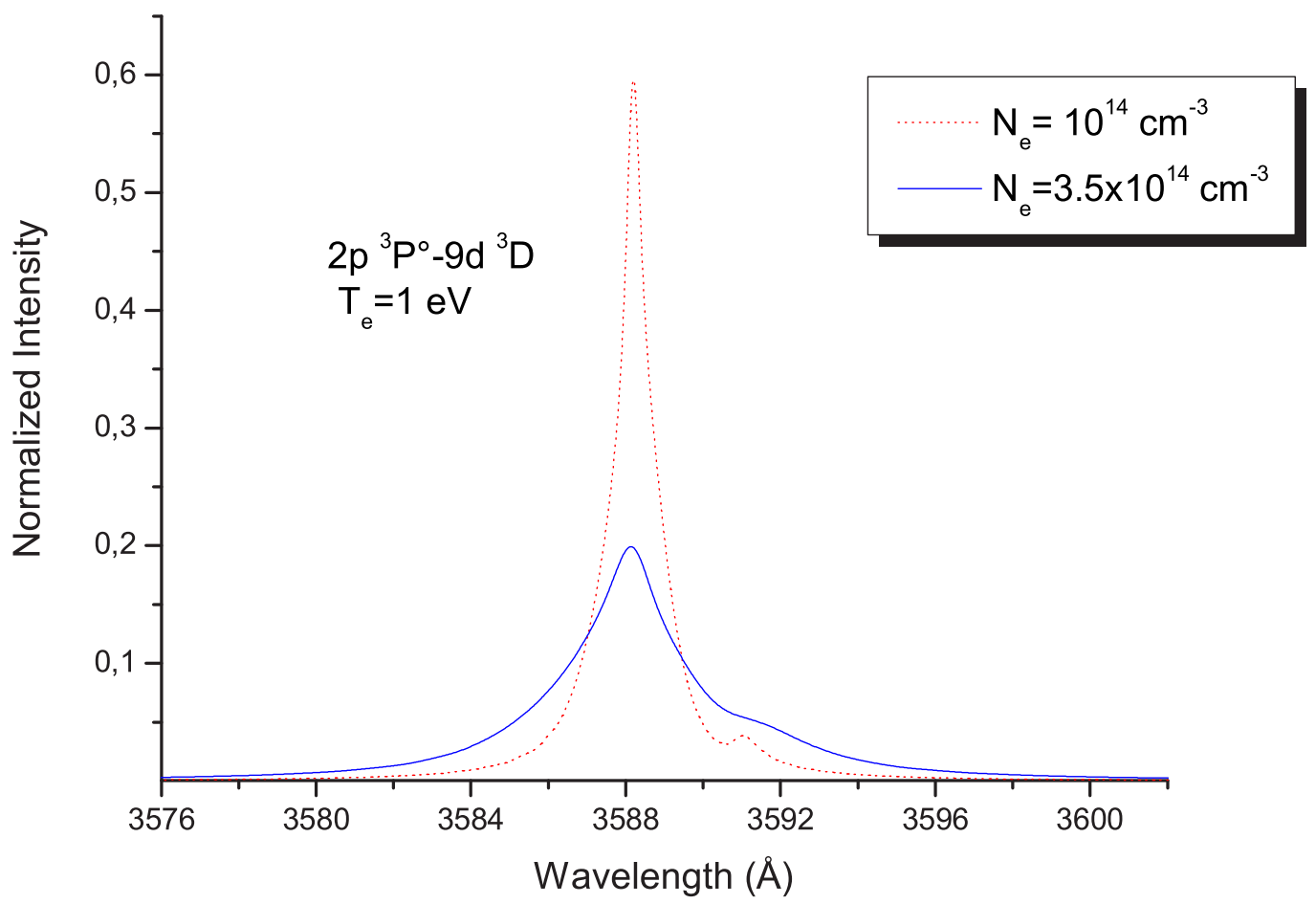

Figure 5. 


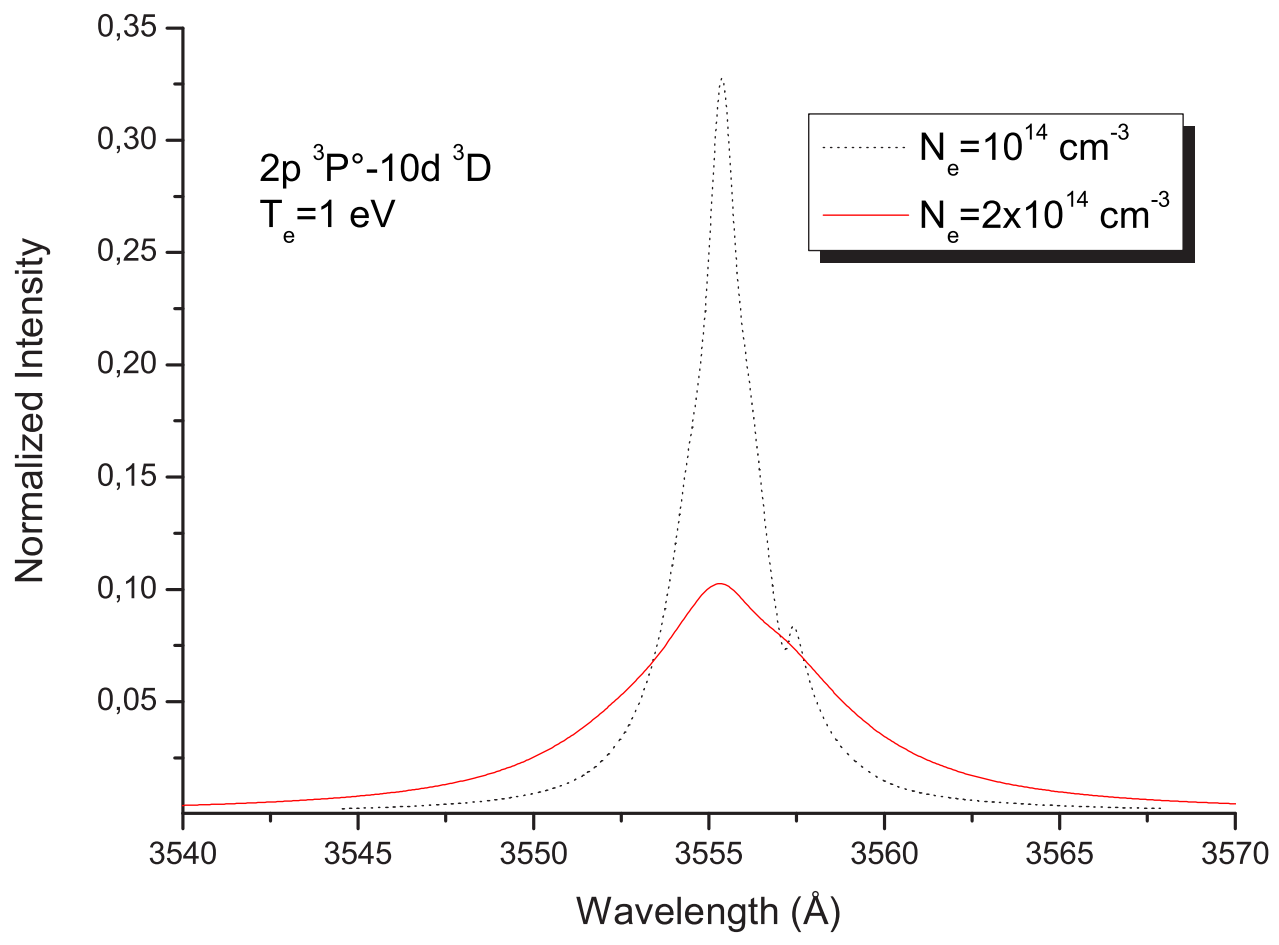

Figure 6. 


\section{References}

[1] G. McCracken et al., Nucl. Fusion 38, 619-629 (1998).

[2] A. Meigs A et al., 25th EPS Conf. on Plasma Phys. Control. Fusion ,Prague, June 29-July 3 (1998), ECA Vol. 22C, P1.017 (http://epsppd.epfl.ch/Praha/WEB/98ICPP_W/B161PR.PDF).

[3] D. Lumma, J. L. Terry and B. Lipschultz, Phys. Plasmas 4, 2555 (1997).

[4] A. Yu. Pigarov et al., Plasma Phys. Control. Fusion 40, 2055-2072 (1998)

[5] U. Wenzel et al., Nucl. Fusion 39, 873-882 (1999).

[6] H. R. Griem Principles of Plasma Spectroscopy Cambridge University Press (1997).

[7] M. Koubiti et al., J. Quant. Spectrosc. Radiat. Transfer 81, 265-273 (2003).

[8] A. S. Kukushkin el al. Europhysics Conference Abstract 33 E, P-4-167 (2009).

[9] M. Koubiti et al., Europhysics Conference Abstract 29 C, P-4.087 (2005).

[10] M. Koubiti et al., Contrib. Plasma Phys. 46, 661-666 (2006).

[11] F. Rosmej et al., 30 ${ }^{\text {th }}$ EPS Conf. on Contr. Fusion and Plasma Phys. St. Petersburg, 7-11 July 2003 ECA Vol. 27A, P-1.176. (http://epsppd.epfl.ch/StPetersburg/PDF/P1_176.PDF)

[12] F. Rosmej et al., 31 ${ }^{\text {st }}$ EPS Conference on Plasma Phys. London, 28 June-2 July 2004 Europhysics Conference Abstracts Vol. 28B, P-4.113. (http://eps2004.clf.rl.ac.uk/pdf/P4_113.pdf)

[13] D. Nishijima et al., J. Nucl. Mater. 290-293, 688-691 (2001)

[14] D. Nishijima et al., Plasma Phys. Control. Fusion 44, 597-610 (2002).

[15] S. Takamura et al., Plasma Sources Sci. Technol. 11, A42-A48 (2002).

[16] N. Ohno et al., Nuclear Fusion 41, 1055-1065 (2001).

[17] H. R. Griem, M. Baranger, A. C. Kolb, G. Oertel, Phys. Rev. 25, 177-195 (1962).

[18] H. R. Griem, Astronom. J. 154, 1111-1122 (1968).

[19] H. A. Gieske, H. R. Griem, Astrophys. J. 157, 963-981 (1969).

[20] M. S. Dimitrijevic, S. Sahal-Bréchot, J. Quant. Spectrosc. Radiat. Transfer 31 301-313 (1984).

[21] M. S. Dimitrijevic, S. Sahal-Bréchot, Astron. Astrophys. 136, 289-298 (1984).

[22] B. Talin et al. J. Quant. Spectrosc. Radiat. Transfer 58, 953-964 (1997).

[23] H. R. Griem, Spectral line broadening by plasmas Academic Press New York \& London (1974).

[24] H. E. White, Introduction to atomic spectra McGraw-Hill Company New York \& London (1934).

[25] http://physics.nist.gov/cgi-bin/AtData/main_asd.

[26] A. Bethe and E. Salpeter, Quantum mechanics of one- and two-electron atoms, Chapter 4, pp 252-264, Springer-Verlag Berlin Heidelberg New York (1957).

[27] M. Goto, in Plasma Polarization Spectroscopy, ed. T. Fujimoto \& A. Iwamae, Springer-Verlag Berlin (2008).

[28] Y. Marandet et al., Europhys. Lett. 69, 531-537 (2005).

[29] J. Rosato et al., Phys. Rev. E. 79, 046408 (2009). 


\section{Figure captions}

Fig.1: Validity of the binary impact approximation for He I $1 \mathrm{~s} 2 \mathrm{p}{ }^{3} P^{\circ}-1$ snd ${ }^{3} \mathrm{D}$ lines ( $n=8-12$ and $n=20$ ) plotted for electron, ion densities and temperatures in the ranges $10^{12} \leq N_{e, i} \leq 10^{15} \mathrm{~cm}^{-3}$ and $T_{e, i} \in[0.03,1000 \mathrm{eV}]$. For electrons, the impact approximation is considered valid above the curves (thin lines) representing $\rho_{w e} / r_{e}=0.1$ for each upper PQN $n$. For ions, only the curves (thick lines) representing the ratios $\rho_{w i} / r_{i}=0.1$ and $\rho_{w i} / r_{i}=1$ corresponding to $n=8$ are plotted. The quasi-static and impact approximations can be used for ions respectively in the regions below the curve $\rho_{w i} / r_{i}=1$ and above the curve $\rho_{w i} / r_{i}=0.1$.

Fig. 2: A schematic representation of the He I transitions from the upper sublevels 1 sns ${ }^{3} S, 1$ snp ${ }^{3} P^{\circ}$, 1 snd ${ }^{3} D, 1 s n f{ }^{3} F^{\circ}$ and $1 s n g{ }^{3} G$ to the lower one $1 s 2 p{ }^{3} P^{\circ}$. Solid and broken arrows represent respectively allowed and forbidden transitions. On the left part of the figure, energy separations between sublevels characterized by $n=8$ are shown.

Fig. 3: Comparison of the dipole reduced matrix elements (in units of the Bohr radius $a_{0}$ ) of the He I $1 s 2 p{ }^{3} P^{\circ}-1 s n d{ }^{3} D$ transitions for $n=4-20$. Data termed "H-approx [26]" that are shown by $(O)$ have been calculated using hydrogenic radial wavefunctions $R_{n l}$ (see text for more details). Values termed "Refs. [14, 25]" and indicated by $(\triangle)$ correspond to conversion of transition probabilities from references $[14,25]$. Note the good agreement between the last data $(\mathrm{O}$ and $\triangle)$.

Fig. 4: Stark profiles of the He I $1 s 2 p^{3} P^{\circ}-1 s 8 d^{3} D$ calculated for a deuterium plasma with an electron temperature of $1 \mathrm{eV}$ and different electron densities. Dotted $(\cdots \cdots)$ and solid $(-)$ lines correspond respectively to $N_{e}=10^{14} \mathrm{~cm}^{-3}$ and $N_{e}=3.5 \times 10^{14} \mathrm{~cm}^{-3}$. The shoulder in the red wing of the line represents a forbidden component.

Fig.5: Same as Fig.4 but for the $1 s 2 p^{3} P^{\circ}-1 s 9 d^{3} D$ line of neutral helium.

Fig.6: Stark profiles of the He I $1 s 2 p^{3} P^{\circ}-1 s 10 d^{3} D$ calculated for a deuterium plasma with an electron temperature of $1 \mathrm{eV}$ and different electron densities. Dashed $(\ldots \ldots)$ and solid ( - ) lines correspond respectively to $N_{e}=10^{14} \mathrm{~cm}^{-3}$ and $N_{e}=2.0 \times 10^{14} \mathrm{~cm}^{-3}$. 